\title{
Neuroanesthesia and Cerebrospinal Protection
}

\author{
Hiroyuki Uchino, Kazuo Ushijima, Yukio Ikeda (Editors). Springer Japan, 2015; Pages: \\ 725. ISBN: 978-4-431-54489-0
}

\author{
Nitin Ahuja, MBBS, MD
}

Received: 12 July 2016/Revised: 8 August 2016/Accepted: 12 August 2016/Published online: 23 August 2016

(C) Canadian Anesthesiologists' Society 2016

The discovery of general anesthesia during the 19th century and advances since then has facilitated a widespread evolution in various surgical specialities, including neurosurgery. The advancement of complex neurosurgical procedures (both in the traditional operating room and in remote areas involving interventional neuroradiology) and the expanded use of intraoperative magnetic resonance imaging have pushed the boundaries of delivering safe, effective anesthesia for neurosurgical interventions. The recent advances in neuroanesthesia have revolved around key considerations to provide effective anesthesia, maximize "neuroprotection," and improve perioperative morbidity and mortality.

The textbook from Uchino and colleagues, Neuroanesthesia and Cerebrospinal Protection, is an effort to amalgamate the complex nature of the processes involved in a neurosurgical patient. The text is divided into 12 broad sections with multiple chapters in each section.

The first three sections give details relevant to neuroanatomy and neurophysiology along with the proposed mechanisms of neuroischemia and neuronal cell death. The next section is dedicated to neuropharmacology, examining the current evidence relating to neurotoxicity caused by commonly used anesthetic drugs. Overall, this section is well covered and nicely describes the current mechanisms and concepts relevant to neuroanesthesia.

The following section is dedicated to various neuromonitoring techniques currently used in neuroanesthesia and neurocritical care settings. In this

N. Ahuja, MBBS, MD ( $\varangle)$

University of Manitoba, Winnipeg, MB, Canada

e-mail: shirinitin@gmail.com section, a detailed and excellent explanation follows, outlining the physiology relevant to neuromonitoring techniques and the key role played by each neuromonitoring modality, including its respective indications, contraindications, and limitations.

Section five addresses some specific issues relevant to neuroanesthesia, including preoperative assessment, patient positioning, neuroimaging, surgical techniques, as well as management of elevated intracranial pressure, cerebral edema, and fluid balance. Overall, although it provides a good selection of topics, this section could have been facilitated by additional editorial input, particularly with respect to language flow (which was somewhat awkward at times) in the section dealing with neurosurgical techniques.

Section six focuses on anesthesia for neurovascular surgery. The first chapter in this section briefly discusses the management of intracranial aneurysms, current grading of subarachnoid hemorrhage, and anesthetic management. The next two chapters address the anesthetic management of extracranial vascular pathologies involving carotid arteries and arteriovenous malformations. Although generally well written, the chapter on intracranial aneurysms could have included more details about the management of intraoperative rupture during clipping and coiling.

Sections seven and eight touch on the anesthesia management of patients with brain tumours, traumatic brain and spinal cord injuries. They include a good discussion of the current evidence for the use of intraoperative neuromonitoring and goals to minimize secondary brain injury.

Section nine covers specific neurosurgical situations. There is a detailed discussion of corrective spine surgery and use of evoked potentials along with the complexity and 
nuance involved in interpreting the results. Further chapters discuss the pituitary, epilepsy, interventional radiology, deep brain stimulation, the pregnant stroke patient, and various neuromuscular diseases. The last chapter in this section discusses massive hemorrhage in general, although with perhaps too much emphasis on local Japanese guidelines and use of simulation as a teaching tool.

The tenth section takes us into the realms of pediatric neuroanesthesia. A well-covered discussion of various situations, such as congenital and traumatic brain injury, is followed by considerations regarding remote location anesthesia, vascular anomalies, and pediatric cardiac surgery. Again, the role of neuromonitoring is discussed extensively, including as a modality to attempt to reduce the postoperative cognitive impairment following pediatric cardiac surgery.

This brings us onto the 11th section of the book labelled "Anesthetic Management: Cardiovascular Surgery and Cerebrospinal Protection." This section goes into considerable detail about the current evidence and our understanding of the aftermath of cardiovascular surgery and cognitive decline. Current approaches to spinal cord protection during aortic surgery and brain protection during adult cardiac surgery are well covered. The last two chapters are dedicated to postoperative cognitive dysfunction seen after cardiac and non-cardiac surgery. This complex section is well written and covers most of the relevant topics in a very readable manner.
The final section of the book addresses "Complications and Other Considerations" in six chapters, the first four of which discuss electrolyte disorders, neurosurgery-specific perioperative complications, pain management, and the current role of hypothermia in brain protection. The section's fifth chapter focuses on extracorporeal cardiopulmonary resuscitation and the role of percutaneous cardiopulmonary support. The last chapter addresses the intricacies of brain death and organ donation with considerable emphasis on the Japanese laws for organ donation.

In summary, this book is a reasonable attempt at a current evidence-based textbook in neuroanesthesia directed toward residents and junior faculty alike. As mentioned above, there are some areas that would benefit from additional editorial input. Another minor criticism is some repetitiveness, the deletion of which would ensure a better flow of sections. Although there is a considerable emphasis on local Japanese content, the perspectives it brings make it a valuable addition to any departmental library. A second edition of the book would be most welcome.

Conflicts of interest None declared.

Editorial responsibility This submission was handled by Dr. Hilary P. Grocott, Editor-in-Chief, Canadian Journal of Anesthesia. 